\title{
Post Varicella Angiopathy as a Cause of Pediatric Stroke: A Case Report
}

\author{
Anju Bala ${ }^{1}$, Parul Bhardwaj', Vipan Garg ${ }^{3}$, Shivbrat ${ }^{4}$ \\ ${ }^{1}$ MD Pediatrics, Civil Hospital Nadaun (H.P.) \\ ${ }^{2}$ MD Internal Medicine, Civil Hospital Dehra (H.P.) \\ ${ }^{3}$ Senior Resident Anesthesia, DRPGMC Kangra at Tanda (H.P.) \\ ${ }^{4}$ Junior Resident ENT, DRPGMC Kangra at Tanda (H.P.) \\ Corresponding Author: Parul Bhardwaj
}

\begin{abstract}
Varicella-zoster virus is a neurotropic herpes virus that contains double stranded DNA. It can cause primary, latent and reactivation infection. Chickenpox is the primary manifestation and result in lifelong latent infection of sensory neurons. Varicella infection is usually self limiting and complications occur mostly in immunocompromised patients. The most common neurological complications following varicella infection are cerebellar ataxia and encephalitis but arterial ischemic stroke in pediatric patient is also significant. Post varicella angiopathy also published under transient cerebral arteriopathy and is nearly always self -limited and may be the result of focal inflammation ${ }^{1}$. Arterial ischemic stroke may occur during the course of varicella infection or one week to several months after the appearance of rash. Here we present a case of 6 years old male who presented with right hemiparesis with right sided facial nerve palsy two months after chickenpox. An MRI was done which was suggestive of hyperintensities in basal ganglia and internal capsule in T-1 weighted MRI. Although the weakness was selflimiting patient was treated with aspirin in addition to supportive measures.
\end{abstract}

Keywords: Arterial ischemic stroke, Post varicella angiopathy, Varicella zoster virus, Middle cerebral artery, Upper motor neuron, Lower motor neuron

\section{INTRODUCTION}

Stroke is an important cause of acquired brain injury in newborn, children and adolescents. Arterial ischemic stroke (AIS) and cerebral sino-venous thrombosis are more common than brain malignancy. One of the leading causes of hemiparetic cerebral palsy is pediatric stroke. Arterial blood reaches the brain via internal carotid and vertebrobasilar arteries covering all the circle of Willis. Strokes most often involve the middle cerebral artery territory. Three main categories of etiology should be considered; arteriopathy, cardiac disease and hematologic disease ${ }^{1}$. Arteriopathy is the leading cause of childhood AIS, present in more than $50 \%$ of children ${ }^{1}$. Arterial ischemic stroke (AIS) during childhood, although relatively rare, but can frequently causes significant long-term morbidity ${ }^{5}$. Varicella is usually a self-limiting disease in childhood. Although mortality and morbidity may be more in immunocompromised individuals, in immunocompetent individuals it usually doesn't lead to any complications ${ }^{3}$. Histopathological examination of the middle cerebral artery (MCA) of a 4-year-old girl who died of VZV vasculopathy revealed granulomatous arteritis with multinucleated giant cells, lymphocytic infiltration, and VZV antigen, primarily in the smooth muscle layer ${ }^{4}$.

\section{CASE REPORT}

6 years old male child presented in opd with complaints of swaying of body to right side, deviation of angle of mouth to 
left side, difficulty in speech and drooling of saliva for 3 days. Weakness was sudden in onset and non-progressive in nature. There was no history fever, vomiting, head trauma, seizures, headache, congenital heart disease, bladder and bowel involvement. Child had history of chicken pox 2 months prior for which child was treated symptomatically at local hospital. There was no history of similar illness in family and neighborhood. Also, there was history of similar episode of weakness 1 month prior which was sudden in onset and weakness improved over 30-60 minutes with no residual hemiparesis. Child was developmentally normal and fully immunized as per national immunization schedule. On examination patient was hemodynamically stable and there were no neurocutaneous markers. There were hyperpigmented spots all over the body. On CNS examination child was conscious and well oriented to time, place and person. On higher mental function examination there was dysarthria. On neurological examination there was right upper motor neuron type of facial nerve palsy in the form of deviation of angle of mouth towards left side and absent nasolabial fold on right side of face. Tone was slightly increased in right upper and lower limb with power of $3 / 5$ in both right upper and lower limbs. Deep tendon reflexes were exaggerated in right upper and lower limb along with upgoing plantar reflex on right side. Child was admitted as a case of pediatric stroke in view of right UMN type facial palsy with right hemiparesis. On routine investigations CBC, coagulation profile, tuberculosis workup was done which was turned out to be normal. ECG and 2D echo were done to rule out cardiac cause of stroke but it was normal. Further investigations were done to look for hypercoagulable state that includes antithrombin III deficiency, homocysteine level, factor $\mathrm{V}$ Leiden mutation, prothrombin gene mutation, but it was normal. After 6 months of weakness protein $\mathrm{C}$, protein $\mathrm{S}$ and factor $\mathrm{V}$ leiden level were done and were normal. On CSF examination there was borderline rise in anti VZV IgM antibodies. On neuroimaging, there were hyper-intensities in T-1 weighted MRI in left basal ganglia and internal capsule suggestive of haemorrhage (a sequence to post varicella angiopathy). CT angiography done was normal. In view of history of chickenpox two months prior to stroke and MRI findings, diagnosis made was transient cerebral arteriopathy due to focal inflammation secondary to varicella infection. The weakness of patient started improving over next two to three days. Patient was started on oral aspirin and continued for six months. Regular physiotherapy was advised and after six months on follow up there was no residual weakness.

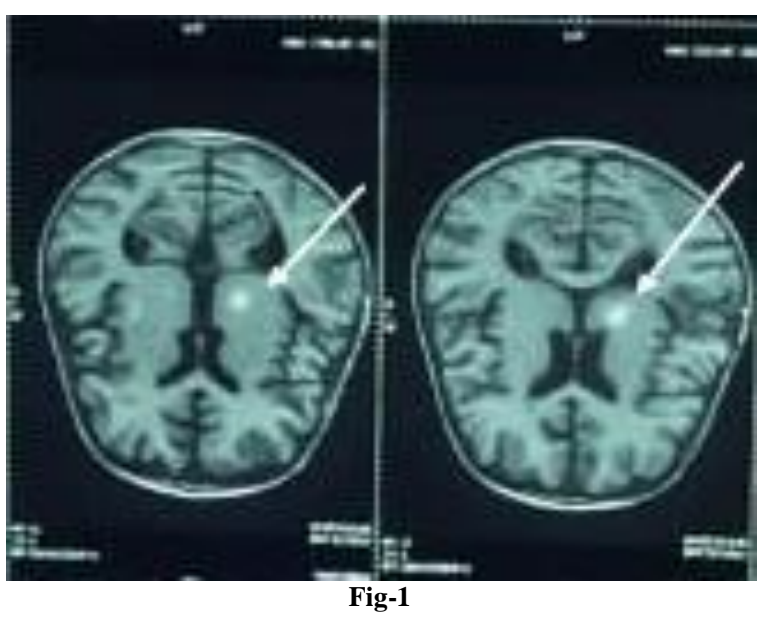

\section{DISCUSSION}

Varicella zoster virus (VZV) is a neurotropic alpha herpes virus. Primary infection, usually in childhood, causes varicella (chickenpox), after which virus become latent in cranial nerve ganglia, dorsal root ganglia and autonomic ganglia along the entire neuraxis ${ }^{2}$. The basic pathogenesis of post varicella angiopathy is that VZV can spread along the ophthalmic branch of trigeminal nerve causing granulomatous angitis with multinucleated cells ${ }^{8} . \mathrm{VZV}$ in the vessel wall may induce a non - cytolytic infection of smooth muscle cells in the media and functional damage of the vascular endothelium resulting in thrombosis and promoting sub endothelial proliferation of smooth muscle cells, 
fibroblasts and collagen resulting in stroke ${ }^{8}$. The most common CT or MRI brain pictures in post varicella angiopathy were infarct of the deeper structures with $89 \%$ involving internal capsule 9 . The spectrum of vascular pathologies secondary to varicella zoster infection includes ischemic infarctions, aneurysm formation, arterial dissections, subarachnoid hemorrhage or intraparenchymal haemorrhage ${ }^{14}$.

The etiology of stroke in pediatric age group is quite different from that of adults. Types of stroke in pediatric age group are arterial and venous thrombosis, intracranial bleeds, embolism and various other conditions. Predisposing conditions for stroke in children include cardiac diseases like congenital heart diseases, arrhythmias, structural valvular heart diseases, bacterial endocarditis, sickle cell disease and occlusive vascular diseases like Moya-Moya disease etc ${ }^{6}$. The underlying mechanism of varicella causing AIS is not clearly understood, although various mechanisms have been suggested and one of the possible explanations is intraneuronal migration of varicella zoster virus from trigeminal ganglion along the trigeminal nerve to cerebral arteries ${ }^{7}$. Recurrence of stroke is more common in the varicella infection associated non-varicella arterial ischemic ${ }^{10}$. Common radiological finding in varicella associated arteriopathy are basal ganglia infarcts and large vessel stenosis. Large artery disease is mainly diagnosed by post stenotic dilatation on MR/CT angiogram, however, direct demonstration of small vessel involvement is difficult as an angiogram and is often negative in this situation ${ }^{15}$. In such cases, the diagnosis is based on cerebral parenchymal changes on MR imaging ${ }^{15}$.

In pediatric age group basal ganglia infarcts may be associated with the history of varicella ranging from $10-50 \%{ }^{11}$. Treatment of varicella associated AIS is mainly supportive. Antiviral therapy and anticoagulants can also be given. Varicella associated stroke has a higher mortality and morbidity in adults ${ }^{12}$. As the survival rate is excellent in children as compared to adults, antiviral therapy and anti-inflammatory therapy may not be given in pediatric patients presenting with AIS associated with varicella infection ${ }^{13}$. Anticoagulant therapy in initial phase of stroke should be considered as this will prevent local extension of the thrombus and chances of embolization. Long term therapy with aspirin should be prescribed to all children suffered from stroke due to varicella infection.

\section{CONCLUSION}

Pediatric stroke is a neurological emergency, although delay in recognition is common as the presenting symptoms in children are difficult to recognize due to lack of proper history, that can lead to poor outcome. Causality of pediatric stroke is different from adult stroke. Post varicella angiopathy is an important cause of pediatric stroke and should be considered in differential diagnosis as it can present as wide range of spectrum on neuroimaging (infarcts, aneurysms, dissections, intraparenchymal haemorrhages). History taking should be good to identify post varicella angiopathy as it can occur after months of varicella infection. Further research need to be done to make proper guidelines for management of pediatric stroke.

\section{Declaration of patient consent:}

The authors certify that they have obtained all appropriate patient consent forms regarding images and other clinical information to be reported in the journal.

Acknowledgement: None

Financial support and sponsorship: Nil.

\section{Conflicts of interest:}

There are no conflicts of interest.

\section{REFERENCES}

1. Dlamini N, deVeber G. Nelson textbook of pediatrics. Philadelphia, PA; Elsevier: 2019 
2. Mahalingam R, Wellish M, Wolf W, et al. Latent varicella-zoster viral DNA in human trigeminal and thoracic ganglia. $\mathrm{N}$ Engl $\mathrm{J}$ Med. 1990;323:627-631.

3. Martin G.Myers, Lawrence R Stanberry and JaneF Seward Varicella Zoster virus In: KliegmanRM, Behrman RE, Jenson HB, editors.NelsonTextbook of Paediatrics. 17th ed. Philadelphia:Saunders; 2004. pp. 1057-1062

4. Berger TM, Caduff JH, Gebbers JO. Fatal varicella-zoster virus antigen-positive giant cell arteritis of the central nervous system. Pediatr Infect Dis J. 2000;19:653-656.

5. deVeber G, MacGregor D, Curtis R, Mayank S. Neurologic outcome in survivors of, childhood arterial ischemic stroke and sinovenous thrombosis. J Child Neurol 2000;15:316-324

6. Michael V. Jhonston Acute Stroke SyndromesIn: Kliegman RM, Behrman RE, Jenson HB,editors.Nelson Textbook of Paediatrics. 17th ed.Philadelphia: Saunders; 2004. pp. 2035-2038.

7. Mayberg MR, Zervas NT, Moskowitz MA.Trigeminal projections to supratentorial pial anddural blood vessels in cats demonstrated byhorseradish peroxidase histochemistry. J CompNeurol 223:46-56, 1984

8. Carvalho KS, Gary BP. Arterial strokes in children. Neurologic Clinics. 2002;20(4): 1079-1100

9. Riela AR, Roach ES. Etiology of stroke in children. Journal of child neurology. 1993; 8(3):201- 220.
10. Hattori H, Higuchi Y, Tsuji M. Recurrent strokes after varicella. Ann Neurol.2000 Jan;47(1):136.

11. Inagaki $M, \quad$ Koeda $T$, Takeshita $K$, Prognosis, and MRI after ischemic stroke of the basal ganglia. Pediatr Neurol. 1992;8:104-108.

12. Gilden DH, Kleinschmidt-DeMasters BK,LaGuardia JJ, Mahalingam R, Cohrs RJ.Neurological complications of the reactivation ofthe varicella-zoster virus. $\mathrm{N}$ Engl J Med.2000;342:635-645.10.

13. Askalan R, Laughlin S, Mayank S, Chan A, MacGregor D, Andrew $M$, et al. Chickenpox and Stroke in Childhood- A Study of Frequency and Causation. Stroke 2001;32:1257-62.

14. Gilden D, Cohrs RJ, Mahalingam R, Nagel MA. Varicella zoster virus vasculopathies: Diverse clinical manifestations, laboratory features, pathogenesis, and treatment. Lancet Neurol. 2009;8:731-40.

15. Harsha KJ, Parameswaran K. Adult postvaricella small vessel vasculopathy mimicking hypertensive basal ganglia haemorrhage with coexisting infarcts. Neurol India 2016; 64: 1323-6.

How to cite this article: Bala A, Bhardwaj P, Garg V et.al. Post varicella angiopathy as a cause of pediatric stroke: a case report. International Journal of Science \& Healthcare Research. 2021; 6(2): 36-39. DOI: https://doi.org/10.52403/ijshr.20210407 